\title{
Dolce sirena e femmina balba: figure femminili nel Purgatorio
}

Douce sirène et femme bègue : figures féminines au Purgatoire

Sweet Mermaid and Stuttering Female: Female Figures in Purgatory

\section{Natascia Tonelli}

\section{(2) OpenEdition}

\section{Journals}

\section{Edizione digitale}

URL: https://journals.openedition.org/cei/9760

DOI: $10.4000 /$ cei.9760

ISSN: 2260-779X

\section{Editore}

UGA Éditions/Université Grenoble Alpes

Edizione cartacea

ISBN: 978-2-37747-304-5

ISSN: $1770-9571$

\section{Notizia bibliografica digitale}

Natascia Tonelli, «Dolce sirena e femmina balba: figure femminili nel Purgatorio», Cahiers d'études italiennes [Online], 33 | 2021, online dal 01 octobre 2021, consultato il 13 octobre 2021. URL: http:// journals.openedition.org/cei/9760 ; DOI: https://doi.org/10.4000/cei.9760

Questo documento è stato generato automaticamente il 13 octobre 2021 


\title{
Dolce sirena e femmina balba: figure femminili nel Purgatorio
}

\author{
Douce sirène et femme bègue : figures féminines au Purgatoire \\ Sweet Mermaid and Stuttering Female: Female Figures in Purgatory
}

Natascia Tonelli

1 Balbuziente, guercia, storpia, senza mani e dilavata nel colore: ecco come appare la cosiddetta femmina balba nel sonno di Dante. Un essere orribile e ripugnante che però, nel corso del sogno narrato nel canto XIX del Purgatorio, subisce una straordinaria, incredibile metamorfosi: il suo parlare si fa soave, il suo corpo meravigliosamente si disviluppa da quel moncherino che si dimostrava essere, quel volto spento e smorto si ravviva e l'incarnato prende colore. Non solo: così trasformata, la nuova immagine di donna inizia un canto di seduzione in cui si dichiara colma di piacere, tanto da saper appagare chiunque. È stata lei, che dice di essere una dolce sirena, ad aver tentato Ulisse nel suo viaggio, è lei che in alto mare ha il potere di disviare i naviganti dalla loro rotta. Nel sogno di Dante improvvisamente appare poi un'altra donna, donna santa e pronta a soccorrerlo, che richiama Virgilio al suo dovere; e così, con gli occhi fitti, cioè traendo ispirazione da essa, evidentemente giunta dal cielo, Virgilio si avvicina alla femmina balba, che ora ha l'aspetto e la loquela di una dolce sirena, e, con gesto repentino e violento, la afferra e squarcia i drappi che la rivestono. Appare quindi il suo ventre, che ne rivela la natura autentica. E non solo quanto all'aspetto esteriore di essere deforme, di femmina balba, ma anche la natura più intima, abominevole, che a quella corrisponde con fisiognomica esemplarità, simboleggiata dal ventre putrido e maleodorante, i cui miasmi hanno la forza di svegliare Dante. Anche questa è una delle figure femminili del Purgatorio, e non certo la meno significativa:

mi venne in sogno una femmina balba,

ne li occhi guercia, e sovra i piè distorta,

con le man monche, e di colore scialba.

Io la mirava; e come 'l sol conforta

le fredde membra che la notte aggrava,

così lo sguardo mio le facea scorta

la lingua, e poscia tutta la drizzava 
in poco d'ora, e lo smarrito volto,

com'amor vuol, così le colorava.

Poi ch'ell'avea 'l parlar così disciolto,

cominciava a cantar sì, che con pena

da lei avrei mio intento rivolto.

«Io son», cantava, «io son dolce serena,

che ' marinari in mezzo mar dismago;

tanto son di piacere a sentir piena!

Io volsi Ulisse del suo cammin vago

al canto mio; e qual meco s'ausa,

rado sen parte; sì tutto l'appago!».

Ancor non era sua bocca richiusa,

quand'una donna apparve santa e presta

lunghesso me per far colei confusa.

"O Virgilio, Virgilio, chi è questa?»,

fieramente dicea; ed el venìa

con li occhi fitti pur in quella onesta.

L'altra prendea, e dinanzi l'apria

fendendo i drappi, e mostravami 'l ventre;

quel mi svegliò col puzzo che n'uscia. (Purg. XIX, 7-33)

2 Sul valore di questo sogno e sulle sue molte suggestioni ci si continua a interrogare: problematica ne è l'interpretazione generale, relativamente al senso che Dante ha voluto conferire all'episodio nel suo complesso, in particolare nell'economia della narrazione del tratto di viaggio purgatoriale e nei rapporti che intrattiene con gli altri due sogni della cantica ${ }^{1}$. Molteplici e disparate sono poi le fonti proposte per l'orrenda immagine femminile'; incerto rimane quale effettivamente sia l'aspetto della sirena evocata da Dante e come si configuri il suo rapporto con Ulisse, l'Ulisse della tradizione omerica ma ancor più con il personaggio della Commedia e riconosciuto alter ego dell'autore; dubbia l'identità della donna scesa dal cielo, se pure sia necessario individuarne una specifica; non chiaro, né sintatticamente né drammaturgicamente, se si tratti di Virgilio o della santa donna a strappare la vesti della sirena per scoprirne il male che occultano.

Ritengo sia particolarmente da evidenziare la più volte sottolineata attiva partecipazione del personaggio Dante alla trasformazione che avviene davanti ai suoi occhi, o meglio, dipendentemente dai suoi occhi: trasformazione che non si darebbe se non vi fosse chi, col suo proprio sguardo, in virtù del sentimento che lo anima, non desse avvio e in certo senso producesse l'incredibile metamorfosi. Il radicale mutamento dell'immagine femminile non avviene infatti motu proprio, come tante delle metamorfosi che incontriamo nella prima cantica del poema: sono gli occhi di chi guarda che agiscono sulla realtà e la deformano, $\mathrm{o}$, in questo caso, la trasformano. È sotto lo sguardo di Dante, a causa del suo sguardo, che ciò che era mostruoso e sgradevole, tanto che l'aspetto lasciava supporre un'intima lordura, corrispondente anche alla bruttura morale, si altera al punto da apparire esattamente il contrario di quello che nella realtà è. Ciò che dà un'apparenza falsa, ingannevole e seducente a una realtà ben diversa sono gli occhi di chi guarda. Dante lo dice e lo ripete: «io la guardavo e il mio sguardo le restituiva una voce piana e, come amor vuole, le ridava vita e colore». In questo caso, gli occhi che vedono ciò che amor impone loro di vedere, travisando evidentemente in modo drammatico la realtà.

4 Dante arriva sulla quarta cornice, là dove si addormenta concependo il secondo sogno del Purgatorio, esausto dall'impegno tanto fisico della risalita quanto intellettuale della 
lunga disquisizione relativa alle varietà e alla qualità dell'amore condotta da Virgilio nei canti precedenti ${ }^{3}$. Discorso sull'amore, ma implicato indissolubilmente con la riflessione su ragione e libero arbitrio, che si trova così «al centro non solo ideale ma anche strutturale del regno dei salvati, centro che coincide poi con quello del poema» ${ }^{4}$. Implicato, anche, con quell'imaginativa ${ }^{5}$ che dà il via al ragionamento nel canto XVII (v. 13: «O imaginativa che ne rube / talvolta sì di fuor, ch'om non s'accorge / perché dintorno suonin mille tube, / chi move te, se 'l senso non ti porge?»), così come è poi implicata con quella specifica facoltà la dinamica di quanto accade nel sogno, come vedremo, sogno introdotto da un vaneggiamento che è da leggersi come termine tecnico che precede l'entrata nella visione (o nei contenuti del sogno) e appunto come tale tecnicamente utilizzato da Dante. È infatti a partire dalla Vita nuova che ne vengono valorizzate le risonanze scientifiche, nello specifico a introdurre e connotare la grande, centrale immaginazione/delirio del capitolo XXIII che preannuncia la morte di Beatrice. Là i termini vano, vaneggiare, immaginare, fantasia, ecc., conoscevano una concentrazione d'uso di rilievo assoluto: e pour cause, visto che il centro del libello verteva proprio sulla promozione del suo autore e personaggio protagonista a individuo connotato da speciali prerogative: perlomeno visionarie e premonitorie, se non già profetiche. È infatti proprio all'altezza della canzone Donna pietosa e del coevo e complementare capitolo in prosa che la contiene quando Dante porta a consapevolezza e a maturazione la progressiva definizione di sé, autore-soggetto delle sue opere, come di chi è dotato di potere divinatorio in virtù, io credo fermamente, di una complessione malinconica dall'aristotelico pedigree che è da qui in poi proprio Dante stesso, coll'attribuirsela, a rivalutare nell'Occidente moderno e che poi Petrarca, assumendola e identificandovisi, rilancia in quello che sarà il suo fortunatissimo futuro di crisma imprescindibile di intellettuali, poeti, filosofi, artisti. L'autoritratto d'artista, che viene delineato in modo circostanziato nel centro drammatico e narrativo della Vita nuova, racconta di colui nel quale, sotto l'attacco di un eccesso di quella malinconia che già prevale nella sua costituzione, la mente si fa "peregrina più da la carne», così che «a le sue visïon quasi è divina» (Purg. IX, 16-18). E questo avviene non solo e non tanto relativamente ai sogni che si fanno presso a la mattina: si configura bensì, per chi sia distinto da tale prevalenza umorale, quale prerogativa specifica e comune in modo fisiologicamente accreditato dai medici (e in origine dall'autorità aristotelica da cui naturalmente e primariamente anch'essi dipendono e la cui formulazione basica e densa nei secoli non fanno che articolare e ampliare):

Nota quare melancolici melius predicunt futura: nescio causam, nisi quia in melancolicis anima est separata a regione corporis et bonorum temporalium, ideo non imaginantur circa talia futura, et ideo plura habent imaginari quam mola [sic] corporis oppressi, et estimant et imaginantur quod non imaginantur alii. Et ideo si tales melancolici essent astronomi [cioè 'astrologi'] circa plura melius possent predicere et iudicare quam alii 6 .

Dante la rivendica per sé a partire appunto dalla Vita nuova ${ }^{7}$. E ad essa si accompagna, nel quadro delineato fin da sempre nella descrizione del carattere malinconico, insieme al genio e alla sua propensione artistica, filosofica e politica, una costitutiva, fisiologica inclinazione alla lussuria: è bene ricordarlo e sottolinearlo, come fa, fin da subito, il problema XXX, 1 ,

Quare omnes precipui circa philosophiam, aut circa curam civilem aut poesim, aut alias artes intenti, videtur melancolici fuisse et morbis colere nigre laborasse? [...] De Bellerofonte etiam dicit Homerus quod solitudinem sectabatur. Sed et alii quamplures cuiusmodi videntur fuisse, ut Empedocles et Plato et Socrates. [...] 
melancolici quamplures luxuriosi sunt, [...] boni ingenii ad discendum, amorosi et cito mobiles ad iram et ad alios anime affectus. [...] Nam et quidam poeta plus valuit in poesi cum a mania caperetur ${ }^{8}$. di immaginazioni e visioni che affollano quel medesimo canto, poi al progressivo vaneggiamento sonnolento del canto XVIII fino alla trasformazione dei pensieri vaganti e vani nel sogno che infine apre il XIX. I tre canti adottano un lessico conforme all'attenta esplorazione dell'incerta condizione fra sonno e veglia, forse la «fenomenologia degli stati mentali» che esercita più fascino su Dante ${ }^{9}$ :

novo pensiero dentro a me si mise,

del qual più altri nacquero e diversi;

e tanto d'uno in altro vaneggiai,

che li occhi per vaghezza ricopersi,

e 'l pensamento in sogno trasmutai. (Purg. XVII, 141-145)

Ma è nuovamente da ribadire come il richiamo al citato capitolo della Vita nuova si attui non solo in termini lessicali, bensì anche grazie alla descrizione delle modalità di passaggio dall'uno all'altro pensiero verso uno stato sempre più incosciente dovuto al vagare incontrollato dei pensamenti. Questi, in modo non del tutto comprensibile, vanno poi ad alimentare di sé, del sentimento che li permeava, il sogno o la visione, o, nel caso di Vita nuova XXIII, il vero e proprio farnetico consegnato alla doppia rappresentazione di prosa e canzone:

Io dico che ne lo nono giorno, sentendome dolere quasi intollerabilemente, a me giunse uno pensero lo quale era de la mia donna. E quando ei pensato alquanto di lei, ed io ritornai pensando a la mia debilitata vita; e veggendo come leggiero era lo suo durare, ancora che sana fosse, sì cominciai a piangere fra me stesso di tanta miseria. Onde, sospirando forte, dicea fra me medesimo: «Di necessitade convene che la gentilissima Beatrice alcuna volta si muoia». E però mi giunse uno sì forte smarrimento, che chiusi li occhi e cominciai a travagliare sì come farnetica persona ed a imaginare in questo modo. (Vita nuova XXIII, 3-4)

E inoltre, la fantasia, che qui, al centro esatto del poema, viene qualificata come alta («Poi piovve dentro a l'alta fantasia / un crucifisso», v. 25), proprio come lo sarà alla sua conclusione («A l'alta fantasia qui mancò possa», Paradiso XXXIII, 142), pare correggere intenzionalmente quella ribadita erroneità, falsità, vanità che la connota in modo ossessivo proprio in Vita nuova XXIII, dove la fantasia è frequentissimamente presente.

9 Dato il quadro delineato, mi trovo in sintonia con quanto sostiene Pertile: se l'«'antica strega' annuncia i tre piaceri carnali che rimangono ancora da espiare sulle tre ultime cornici della montagna - l'avarizia, la gola, la lussuria - e il modo in cui l'uomo si slega da essi», «di questi tre peccati la lussuria è l'unico in cui Dante si dimostri fortemente e personalmente implicato» ${ }^{10}$. Insisterei in questa direzione, fino a sostenere che è proprio questo specifico peccato che la visione si fa effettivamente carico di allegorizzare. La trasformazione di un essere mostruoso in sirena ammaliatrice, dimostrazione in corpore vili di quella specifica propensione al lasciarsi sedurre da false immagini di bene di cui Beatrice accuserà di lì a poco Dante, è da ricondursi a un abbaglio d'amore, uno sbaglio dovuto all'amore, a quell'amore che è desiderio e lussuria: concetti e vizi che la sirena, animale/donna, essere per eccellenza dalla doppia natura (nella combinazione di pesce o uccello che sia), bene incarna e usualmente rappresenta nell'universo simbolico del medioevo. Oltre a rendere proverbialmente ciechi, la signoria esercitata da Amore sull'intelletto e sui sensi ha il potere di 
trasformare in bello e seducente, degno di venerazione, ciò che bello non è affatto $\mathrm{e}$ che, anzi, nasconde un potenziale pericolo di degrado e di degenerazione morale.

La donna, il corpo della donna, tradizionalmente, è veicolo di un traviamento che, come si sa, viene esercitato attraverso l'attrazione della bellezza: ab Eva principium, si potrebbe dire. Il simbolo della sirena anche qui sta a rappresentarlo icasticamente, come già nei bestiari moralizzati, fra cui il Bestiario Toscano:

La serena si è una criatura molto nova, ché elle sono di tre nature. L'una si è meço pescie e meça facta a similitudine de femena. L'altra si è meço uccello e meço femena. L'altra si è meço como cavallo e meço como femena. Quella che è [meço] pescie si à sì dolce canto che qualunque homo l'ode si è misteri che sse lli apressime. Odendo l'omo questa voce, sì si adormenta, e quando ella lo vede adormentato sì li viene sopra e uccidelo. Quella che è meço cavallo, sì sona una tromba che simigliantemente è sì dolce che occide l'omo in quella medesma maniera. Quella ch'è meço uccello si fa uno sono d'arpa di tale mainera che simigliantemente è homo tradito e morto. Questa serena potemo noi appellare le femene che sono di bona conversatione, che ingannano li homini li quali s'inamorano di loro carnalmente, che per qualunqua chagione li homoni s'inamorano di loro o per beleçça di corpo o per vista che ella li faccia o per paraule inganevile ch'ella dice, si può tenere morto sì como collui cui la serena ne inganna; ché chi di folle amore è preso bene po dire che sia morto in tutti l'altri suoi facti. Sì como dice in uno luogo: Quando l'omo è d'amore preso, arivato è a mal porto; allora non è in sua balìa, e chi per sua mala ventura morisse in quello stato puote dire che sia morto in anima e in corpo ${ }^{11}$.

È perché Dante proprio udendo le sirene sia più forte che il sogno gli mostra in modo esplicito i meccanismi dell'auto illusione che l'amore per malo obietto e per troppo [...] di vigore induce nell'uomo, che ha indotto in lui, Dante, dopo la morte di Beatrice, come dalla stessa donna gli sarà definitivamente smascherato a fondo nel paradiso terrestre. È come se in questo sogno assistessimo a una sorta di piccola rappresentazione allegorica di ciò che significa lasciarsi adescare dall'amore, una drammatizzazione che si svolge in sogno di quel che produce l'amore quando agisce sulla facoltà più nobile dell'individuo annullandola, quando l'amore sia totalmente dissociato dalla ragione.

Non certo tutti i tipi d'amore determinano tale devianza della mente e dell'intelligenza, tale rischio potenziale per la mente e per il corpo. È solo l'amore eccessivo, smisurato, oltre misura di natura, come lo definisce Cavalcanti, e che ha fra l'altro rilevanza clinica, perché mette a repentaglio la salute fisica dell'amante e ne annulla la lucidità mentale: in quanto ne subordina la ragione all'impulso del desiderio. L'amore folle del Bestiario sopra citato, quello stesso che caratterizza i lussuriosi, coloro che la ragione sommettono al talento, nei confronti dei quali la condanna etica è corroborata dalla scienza medica più all'avanguardia a quel tempo. 0 meglio, la scienza descrive come la facoltà estimativa dell'individuo preda della passione trasformi ciò che vede in qualcosa che con la verità non ha alcun rapporto, e perciò si pone l'obiettivo di trovare cure per contenere o rimediare alla devianza della ragione, cioè della facoltà del giudizio, la virtus che ne risulta maggiormente intaccata, poiché, in questi casi, anche dal punto di vista della scienza la ragione è sommessa al talento: «corruptum est iudicium rationis», «sed concupiscentia non quiescit intantum».

13 Secondo la concezione patologica dell'amore che la medicina aveva contribuito a diffondere, come risulta dai diffusissimi trattati medici dal Canon di Avicenna in poi, e la cui produzione si fa particolarmente intensa negli ultimi decenni del Duecento, l'errore di giudizio che sta all'origine della fissazione maniacale dell'immagine dell'essere amato nella mente dell'amante modifica la realtà e falsa la percezione, al 
punto che l'amante ritiene l'amata l'essere più bello e affascinante al mondo. 0 , per essere più pedestri e prosaici, nella formula che ripete in un fortunatissimo trattato medico un luminare a Montpellier contemporaneo di Dante, "quisquis amat ranam, ranam putat esse Dianam»: «se sei innamorato, ti sembrerà Diana anche una rana...». Bernardo di Gordon, che pubblica il suo Lilium medicinae nel 1305, nel capitolo dedicato all'amore, De amore qui hereos dicitur (da cui anche le citazioni precedenti), illustra molto bene quali siano le procedure dell'autoinganno. E poiché «Causa huius passionis est corruptio extimative propter formam et figuram fortiter affixam», il primo obiettivo del medico sarà quello di ricondurre a retto giudizio la facoltà estimativa, ripristinandone la funzionalità: passaggio decisivo perché il paziente possa recuperare, con il senno, anche la salute. Ciò implica la preliminare rimozione della falsa immaginazione in cui questi si trova immerso: «Si [patiens] est obediens, removeatur ab illa falsa ymaginatione $a b$ aliquo viro quem timeat, de quo verecundet, cum verbis et admonitionibus ostendendo pericula seculi, diem iudicii et gaudia paradisi $\rangle^{12}$.

Un uomo autorevole, la cui voce abbia peso e importanza presso l'innamorato, gli ricordi i pericoli del mondo e a cosa conduca il cedervi per il giorno del giudizio che verrà, gli dica delle gioie del paradiso. La parola suasoria di un maestro può avere efficacia però solo per determinati pazienti non troppo protervi. L'asprezza dei rimedi si deve così acuire proporzionalmente all'ostinazione, fino a giungere a individuare la necessità, anche da parte di un magister, accademico di vaglia e famoso scienziato come il Gordon, là dove la sua teoria non riesca a provvedere, di fare ricorso alle risorse delle snobbatissime pratiche popolari, alla saggezza di quelle donne che, parallelamente alla medicina ufficiale, e da questa usualmente disprezzate, si occupavano tradizionalmente della salute di vecchi, donne e bambini. Dunque, esaurito ogni altro tentativo di cura, non resta che il ricorso all'anula:

Finaliter autem cum aliud consilium non habemus, imploremus auxilium vetularum ut ipsam dehonestent et diffament quantum possunt: ipsae enim habent artem sagacem ad hoc plus quam viri; tamen dicit Avicenna quod aliqui sunt, qui gaudent in audiendo foetida et illicita. Quaeratur igitur vetula turpissima in aspectu cum magnis dentibus, et barba, et cum turpi et vili habitu, et quod portet subtus gremium pannum menstruatum, et adveniens philocaptum quod incipiat dehonestare amasiam suam, dicendo quomodo est tignosa et ebriosa, et quod mingit in lecto, et quod est epileptica, et impudica, et quod in corpore suo sunt excrescentiae enormes cum foetore anhelitus, et aliis omnibus enormibus, in quibus vetulae sunt edoctae. Si autem ex his persuasionibus nolit dimittere, subito extrahat pannum menstruatum coram facie, poetando, dicendo, clamando: talis est amica tua, talis. Et si ex his non dimiserit, iam non est homo, sed diabolus incarnatus. Fatuitas igitur sua, ulterius secum fit in perditione ${ }^{13}$.

Nella generale impostazione teorica per la quale contraria contrariis curari, così si esprime Pietro Ispano nella prima delle due redazioni delle Questiones super Viaticum (Questiones super Viaticum [red. B], rr. 198-202): «mulieres turpes contrariantur amasie patientis amorem hereos, cum illa sit pulcra secundum estimationem ipsius. Ergo visus turpium mulierum competit in amore hereos ${ }^{14} 》$.

Utile dunque risulta, nei casi di amore hereos, far vedere mulieres turpes, tentare la terapia del brutto che sappia restituire il paziente a un sano principio di realtà. Ma in Bernardo di Gordon c'è di più: la pantomima pretende di dimostrare non solo in re cosa sta dietro l'allucinazione di bellezza che ammala il paziente, ma, in una felice coessenza di terapia e terapeuta, la sagace arte della vetula turpissima unisce parole e gesti eloquenti che accrescano con efficacia l'effetto salutare già dovuto al suo proprio 
aspetto. La climax della scena consiste nell'estrarre dal grembo, nascosto sotto la vile veste, un panno mestruato che rivela quella che è anche la lordura intima del corpo femminile, accompagnata, possiamo immaginare, «cum foetore anhelitus».

Il sogno del Purgatorio sembra mettere in scena, drammatizzare, propriamente, quanto è contenuto in questo del Gordon, assai articolato, come in tanti altri capitoli medici del tempo dedicati all'amore. Ne ampia anzi la trattazione alla fase precedente la rilevazione e la conclamazione dello stato patologico di stretta competenza medica, rappresentando in modo straordinario l'intero processo in atto a parte subiecti, dal punto di vista di un infatuato competentissimo e lucido - pur nel delirio che descrive -, fin dal momento iniziale: dall'occhio che, come amore vuol, altera la raccapricciante figura femminile in virtù dell'estimativa corrotta; alla falsa immagine di bene creata, allucinazione dalla quale l'estimativa fa dipendere l'appagamento di ogni desiderio; fino all'intervento autorevole del maestro, ragione vs illusione dei sensi, che, manu medica, dalla parte della ragione e della morale, restituisce a vista e olfatto la corretta percezione, la realtà che sta dietro l'immagine del desiderio. Dopo l'ampio intervento sull'amore dei canti che precedono, abbiamo nel XIX, sotto forma allegorica di visione, un vero e proprio trattato dantesco in versi sulla patologia dell'amore passione, cove sono ben declinate causae e curae. E chi interviene con gesto repentino a squarciare le vesti che coprono l'orrido ventre per mostrarlo proprio a Dante con fine educativo / curativo («dinanzi l'apria / fendendo i drappi, e mostravami 'l ventre», vv.31-32), non sarà la 'santa donna' scesa dal cielo (presumo Beatrice stessa), ma proprio Virgilio, da lei richiamato al preciso compito che gli è stato assegnato fin dall'inizio del viaggio di incarnare al grado supremo la ragione umana, la sua potente capacità di capire e scoprire dove si trovino il bene e il male, di consentire all'uomo, nella sua personale libertà, di sceglierli liberamente, a patto, appunto, che non si lasci ottenebrare dalla seduzione dei sensi indotta da un'estimativa corrotta. Il maestro che ha fin lì discettato sulle questioni dell'amore, nel sogno agisce anche praticamente, rimuovendo il malo obietto, quale ideale incarnazione di chi con autorità possa svelarne i mascherati orrori. Come consigliava fra gli altri il grande medico e filosofo Arnaldo da Villanova in uno dei suoi interventi sull'amore cosiddetto 'heroico':

Distrahit quoque mens cogitantis heroyci duro ac reprehensivo colloquio verende persone: odium et displicentia rei desiderate procurant videre aut audire deformitates turpitudines ac utilitates eius atque narranda. Tales sunt eligende persone que caute faciant esse credibile quod narrabunt ${ }^{15}$.

Una linea di lettura in prospettiva medica viene dunque a mio parere ad unirsi al generale ambito culturale di pertinenza delle immagini muliebri della visione dantesca, facendone ovviamente essa stessa parte: la peculiare e pervasiva misoginia (o ginofobia) connaturata al cristianesimo a far data dal peccato di Eva che si traduce nella donna diaboli ianua di Tertulliano e che si propaga poi, in letteratura, combinandosi con la linea satirica della tradizione classica, fino alla vedova del Corbaccio (che sotto una artefatta bellezza cela nefandezze ridicolizzabili e moralmente pericolose a un tempo e che conducono direttamente alla dannazione eterna del porcile di Venere) o agli spunti di Augustinus nel Secretum. Il tema della seduzione erotica e peccaminosa del corpo femminile ammantato di contro il corpo di Laura falsa belleezza e del disvelamento educativo di ciò che una fuorviante apparenza occulta è particolarmente sviluppato nel contesto monastico; da esso infatti provengono i casi di exempla di tentazioni diaboliche 'sub speciae foeminarum' che i commentatori danteschi hanno messo di volta in volta in rilievo al proposito della femmina balba / 
dolce sirena: a partire da quella che mi pare sempre assai pertinente proposta da Toffanin relativa alla foetida Aetiopissa ${ }^{16}$. A questo ricco repertorio di narrazioni medievali, cui si sono peraltro aggiunte svariate altre ipotesi di fonti (folkloriche, bibliche, pseudo ovidiane, boeziane $)^{17}$, ritengo possa accostarsi anche una delle visioni di Ildegarda di Bingen contenute nel Liber Scivias, Undecima visio tertiae partis:

Deinde vidi... et illa muliebris imago quam ante altare quod est ante oculos Dei prius conspexeram, nunc etiam mihi ibidem iterum ostensa est, ita tamen quod eam modo etiam ab umbilico uentris sui deorsum uiderem. Nam ab umbilico suo usque ad locum illum ubi mulier cognoscitur varias et squamosas maculas habebat. In eodem autem loco muliebris cognitionis monstruosum et nigerrimum caput apparuit, igneos oculos et aures ut aures asini et nares et os ut nares et ut os leonis habens, ac magno hiatu frendens, et uelut ferreos ac horribiles dentes horribiliter acuens.

Sed ab eodem capite usque ad genua sua eadem imago alba et rubea et uelut multa contritione tunsa erat, ab ipsis autem genibus usque ad duas zonas, quae per transuersum superius tangentes talum albae uidebantur, sanguinea apparebat. Et ecce idem monstruosum caput se tanto fragore a loco suo dissoluit, quod omnis eadem muliebris imago in omnibus membris suis inde concuteretur.

Sed et uelut magna massa multi stercoris ipsi capiti coniuncta est, unde illud super quendam montem se eleuans altitudinem caelorum ascendere tentauit. Et ecce uelut ictus tonitrui repente ueniens idem caput tanta fortitudine repercussit, quod et de monte eodem cecidit et quod spiritum suum in mortem emisit. Unde subito foetens nebula ipsum montem totum comprehendit ${ }^{18}$.

19 Da che riluceva al cospetto di Dio, una volta che la si osservi dall'ombelico in giù, la donna protagonista della visione rivela un ventre coperto di macchie e squame; dove soprattutto è presente una testa mostruosa e nerissima, a sua volta con caratteristiche di asino e di leone, che digrigna orribilmente i denti. Staccandosi poi dal ventre della donna con grande fragore, la testa trascina con sé un'enorme massa di sterco, cerca di risalire su un monte verso l'alto dei cieli. Ma un colpo di tuono la colpisce facendole esalare l'ultimo respiro, così che una nube puzzolente circonda tutto il monte.

Quanto descritto è poi rappresentato in una delle splendide immagini del codice di Rupertsberg (Wiesbaden 1: di cui resta, dalla sua distruzione nel corso di un bombardamento nel 1945, oltre a documentazione fotografica, una copia miniata del 1927), il cui programma iconografico è da attribuirsi alla medesima Ildegarda ${ }^{19}$. La miniatura a tutta pagina, nel riquadro maggiore che contiene la porzione di nostro interesse, mostra la donna con aspetto regale e regalmente addobbata fino alla vita, priva di veste dalla cintura in giù a scoprire lo scabroso e fetido portato del suo grembo. Il contesto, inoltre, è quello visionario e mistico di chi ha esperito, e invita a esperire, le 'vie della luce' (se Scivias sia, come pare, l'acronimo di 'Scito vias lucis'). 
Figura 1. - Ildegarda di Bingen, Liber Scivias, Undecima visio tertiae partis (copia del codice Hs1 Rupertsberg).

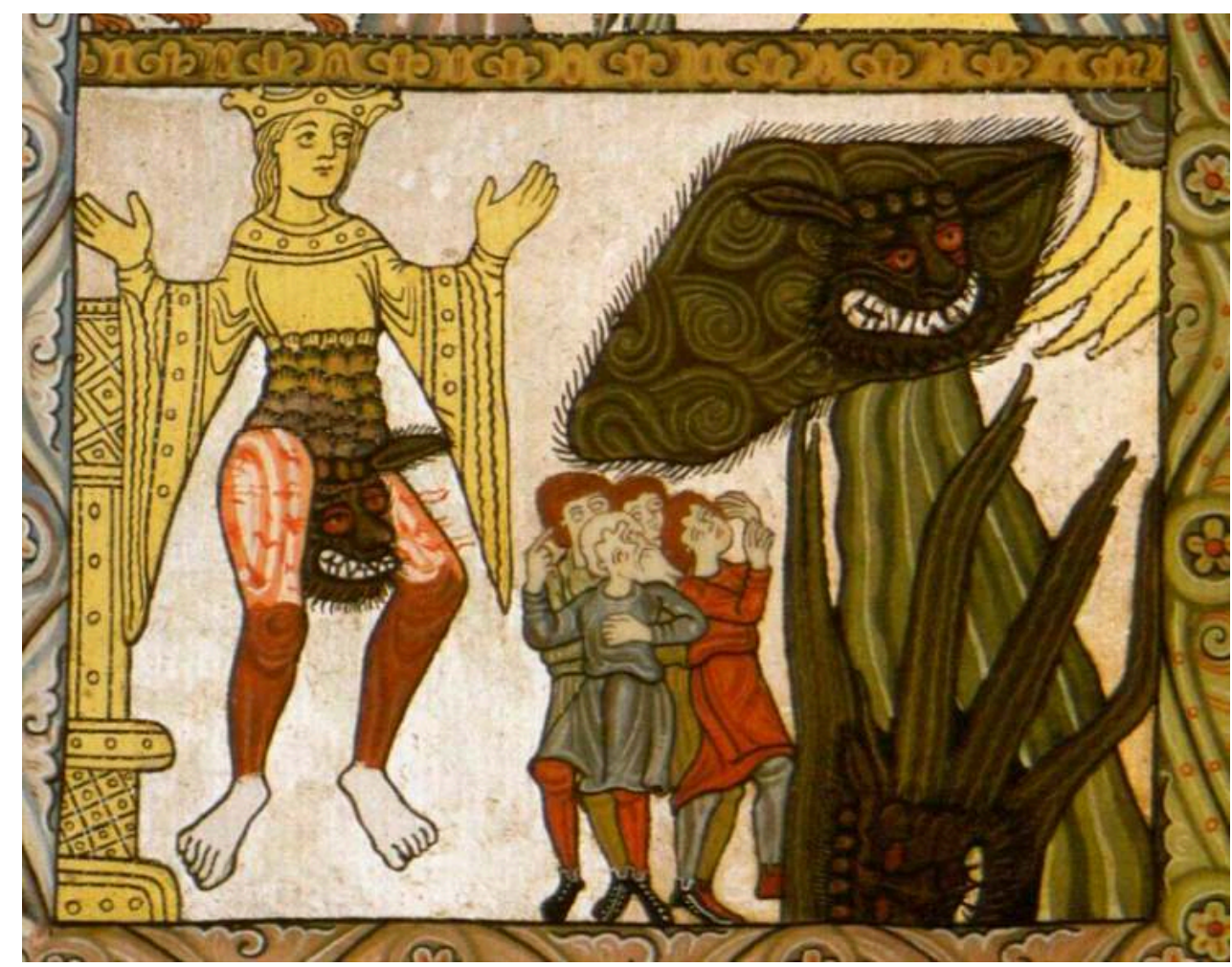

21 Risulta ancora non accertata la conoscenza della scienziata, musicista, medica e profetessa Ildegarda di Bingen da parte di Dante, benché recenti proposte di contatti testuali a mio parere sollecitino a riconsiderare come ben plausibile il rapporto, data anche la notevole diffusione delle sue opere quale è testimoniata dai numerosi codici superstiti ${ }^{20}$. Tuttavia, propongo qui di valutare la visione e la miniatura di Ildegarda non già in funzione di una relazione privilegiata col testo dantesco, quanto a ulteriore corredo di un ampio, compatto contesto culturale di cui anche il sogno purgatoriale viene a far parte e che anzi, da par suo, Dante rende più intenso e rilancia. Se infatti la femmina balba che si trasforma in essere seducente, e poi l'essere seducente che, a penetrarne l'intima sostanza, rivela la sua identità di essere orrendo (visto che presumibilmente il ventre scoperto dal gesto di Virgilio è solo la prima fase di un processo di restituzione dell'immagine della sirena alla realtà di donna deforme e balbuziente, una retro trasformazione - da carrozza a zucca, per intenderci), se questa doppia metamorfosi realtà-illusione-realtà sta a dimostrare - oltre agli abbagli dell'amore - anche la natura doppia, infida, tentatrice, che induce al peccato, dei 'mali oggetti' dell'amore, cioè delle 'pure femmine', la sirena è qui utilizzata da Dante (come lo sarà poi da Beatrice, che rinvia credo esplicitamente a questo passo in Purg. XXXI, 43-45), a confermare e rafforzare la portata della rappresentazione educativa contenuta nel sogno. Questa infatti ne è la funzione: sorta di visibile parlare 'personalizzato', ad uso del Dante penitente. La sirena stessa è già infatti un mostro ibrido, dei tanti della Commedia, fra donna e uccello (come le arpie, con proporzioni mutate), secondo la credenza più diffusa nel Medio Evo; ovvero fra donna e pesce (quasi serpente ${ }^{21}$, incarnazione edenica del demonio ${ }^{22}$. È cioè figura per eccellenza della doppiezza di un femminile perturbante che con una parte di sé ammalia e seduce e con l'altra spaventa e respinge, doppiezza che mi pare rappresentata con grande forza iconica dalla visio e 
in particolare dalla miniatura della mulier di Ildegarda (pur se di ben altro tratti, come la stessa Ildegarda specifica nell'interpretare la visione). L'entità che si autoproclama dolce serena è una sorta di mise en abyme o di concentrato in una sola figura delle due donne che si avvicendano nella scena della metamorfosi, una orribile e l'altra, apparentemente, bellissima. A sua volta, la doppia natura della sirena credo possa assumersi come plausibile chiave di lettura di molti personaggi muliebri della Commedia, e segnatamente del Purgatorio. La traccia da seguire - che riservo ad un prossimo approfondimento - fa tappa nell'autonominazione di Sapia, «Savia non fui, avegna che Sapia fossi chiamata», il cui stesso nome racchiude e implica l'inganno; e passa anche attraverso la figura di Alagia, evocata proprio in questo canto XIX:

Nepote ho io di là c'ha nome Alagia,

buona da sé, pur che la nostra casa

non faccia lei per essempro malvagia. (vv. 142-144)

Papa Adriano V è senz'altro portavoce di un omaggio alla donna in quanto consorte del Malaspina generoso ospite di Dante, ma l'omaggio è realizzato attraverso una formula piuttosto ambigua, che non pregiudica il permanere di un inquietante beneficio del dubbio: quale sarà il futuro disvelarsi-rivelarsi di un femminile che, per quanto dotato di buona natura, non si è mai certi di cosa possa nascondere o in cosa possa evolvere se, nel caso, messo alla prova da cattivi esempi? Proprio all'altro capo del canto risulta infatti emblematicamente incarnato in femmine balbe che sanno celarsi dietro false apparenze e in mostri ibridi e traditori: a gettare le basi per una ben comprensibile, generale preoccupazione.

\section{NOTE}

1. Per una bella e importante lettura di tutti e tre i sogni purgatoriali, si veda G. Barucci, «Simile a quel che talvolta si sogna». I sogni del Purgatorio dantesco, Firenze, Le Lettere, 2012.

2. Si veda un ampio panorama degli studi in T. Caligiure, La «femmina balba» $e$ la «dolce serena», «Rivista di studi danteschi», vol. IV, $\mathrm{n}^{\circ} 2$ 2, 2004, pp. 333-366. Trovo estremamente suggestiva la proposta recente di E. Rebuffat, Nell'ora più fredda. Un'altra idea della femmina balba (Purg., XIX 1-33), «Rivista di studi danteschi», vol. XVIII, n² 2, 2018, pp. 278-319, che propone un ibrido donna/ serpente per la inquietante 'femmina' dantesca, sul modello della diffusa raffigurazione del demonio quale serpente tentatore edenico con volto e busto di donna. Sono forti le ragioni 'naturalistiche' che Rebuffat individua nel testo dantesco, combinate inoltre con l'avantesto ovidiano della metamorfosi di Cadmo (dai moncherini alla similitudine delle fredde membra, che si risvegliano allo sguardo dell'osservatore come al calore del sole, all'assenza di colore), che vengono a configurare la femmina balba come «fase intermedia di una metamorfosi da serpente a sirena» (p. 302). In questa lettura troverebbe spiegazione anche la caratteristica divenuta antonomastica della donna, la sua «voce [...] / a parole formar disconvenevole» (Inf. XXIV, 65-66), come già riferita ai protagonisti delle metamorfosi infernali, che rispecchierebbe così la sua non ancora compiuta trasformazione.

3. Ha sottolineato la continuità del canto XIX col precedente R. Hollander, The Women of Purgatorio: Dreams, Voyages, Prophecies: «The poetic source of this dream lies in the preceding canto» (in Id, Allegory in Dante's Commedia, Princeton, Princeton University Press, 1969, 
pp. 136-191, a p. 139), e poi ancora: «The Siren comes, not only from Dante's reading in the Ulysses literature, but from the text of Virgil's discourse on love» (p. 140).

4. Così A. M. Chiavacci Leonardi, in Dante, Purgatorio, Milano, Mondadori, 1994, p. 491.

5. Si veda il volume del Lessico Intellettuale Europeo dedicato a Phantasia-Imaginatio, Atti del V Colloquio internazionale (Roma, 9-11 gennaio 1986), a cura di M. Fattori e M. Bianchi, Roma, Edizioni dell'Ateneo, 1988, in part. i saggi di R. Busa, De phantasia et imaginatione iuxta S. Thomam, pp. 135-152 e J. Hamesse, Imaginatio et phantasia chez les auteurs philosophiques $d u 12^{e}$ et du $13^{e}$ siècle, pp. 153-184; da ricordare anche M. D. Chenu, Imaginatio. Note de lexicographie médiévale [1946], in Id., Studi di lessicografia filosofica medievale, a cura di G. Spinosa, Firenze, Olschki, 2001, pp. 127-136. I. Baldelli, Visione, immaginazione e fantasia nella Vita nuova (in I sogni nel Medioevo, Seminario internazionale, Roma, 2-4 ottobre 1983, a cura di T. Gregory, Roma, Edizioni dell'Ateneo, 1985, pp. 1-10) insisteva opportunamente sull'uso scientificamente competente del lessico 'visionario'.

6. Gerardi de Solo, Commentum super nonum Almansori, Venetiis, per Bonetum Locatellum, 1505, c. $31 \mathrm{v}$.

7. Per un quadro completo di questa proposta di lettura del capitolo della Vita nuova, v. il mio Fisiologia della passione. Poesia d'amore e medicina da Cavalcanti a Boccaccio, Firenze, SISMEL Edizioni del Galluzzo, 2015, part. capp. 3 e 4; per il processo di autoconsapevolezza fisiologicovisionaria compiuto da Dante, la voce Medicine, in M. Gragnolati, E. Lombardi e F. Southerdern (a cura di), The Oxford Handbook of Dante, Oxford, Oxford University Press, 2021, pp. 227-241.

8. Lo cito dalla traduzione di Davide di Dinant, De effectibus colere nigre, in I testi di David di Dinant: filosofia della natura e metafisica a confronto col pensiero antico, introduzione e edizione dei testi a cura di E. Casadei, Spoleto, Fondazione centro italiano di studi sull'Alto Medioevo, 2008, pp. 189-288, a pp. 189 e 190.

9. Come evidenzia Robert Hollander nel suo commento ai primi versi del canto XVII, «Esempi di lessico connesso all'idea di 'immagine' si incontrano trentotto volte nel poema. Poco più di un ottavo dei casi occupa, dunque, 1/300 del poema» (Dante Alighieri, Commedia, con il commento di R. Hollander, traduzione di S. Marchesi, Firenze, Olschki, 2011, vol. II, p. 143; la citazione a testo ivi, p. 146). Sulle caratteristiche negative dello specifico tipo di fantasia qui attivo, si veda R. Pinto, La femmina balba e la fantasia proterva (Dante, Purg. XIX 1-33), in P. Amalfitano (a cura di), Il piacere del male. Le rappresentazioni letterarie di un'antinomia morale (1500-2000), t. I: Dal Cinquecento al Settecento, Pisa, Pacini, 2017, pp. 91-108.

10. Nella bella lettura di Lino Pertile, Il cigno e la sirena. Lettura del canto XIX del Purgatorio, in B. Quadrio (a cura di), Esperimenti danteschi: Purgatorio 2009, Genova, Marietti, 2010, pp. 175-196 (<https://dash.harvard.edu/bitstream/handle/1/33723554/cignosirena-1.pdf?

sequence=3\&isAllowed=y>, p. 6).

11. Già in Pertile, cit., < https://dash.harvard.edu/bitstream/handle/1/33723554/ cignosirena-1.pdf?sequence=3\&isAllowed=y>, p. 4.

12. Bernardi Gordonii Opus lilium medicinae, Lugduni, Rovillio, 1551; il capitolo De amore qui hereos dicitur è il XX della II parte.

13. Lilium medicinae, part. II, cap. XX, p. 218. A Paolo Cherchi (Per la "femmina balba", "Quaderni d'Italianistica» vol. VI, $\mathrm{n}^{\circ} 2$, 1985, pp. 228-232) si deve l'accostamento fra questo paragrafo del testo di Bernardo e il sogno del XIX del Purgatorio. Per una discussione del passo e per una giustificazione della lettera del testo che differisce in qualche decisivo punto da quello letto da Cherchi, e quindi dalla sua interpretazione, v. Fisiologia della passione, cit., pp. 203-204.

14. Pietro Ispano, Questiones super Viaticum, in M. F. Wack, Lovesickness in the Middle Ages. The Viaticum and Its Commentaries, Philadelphia, University of Pennsylvania Press, 1990, pp. 212-251.

15. Arnaldi de Villanova De parte operativa in Opera nuperrime revisa, Lugduni, Huyon, 1520, cc. $123-130$, c. 129 r.

16. G. Toffanin, La «foetida Aetiopissa» e la «Femmina balba», in Id., Sette interpretazioni dantesche, Napoli / Bari, Libr. Scientifica Editrice, 1947, pp. 29-34. 
17. Penso in particolare agli apporti dei già citt. Barucci, "Simile a quel che talvolta si sogna"..., in part. pp. 144-153 e Caligiure, La «femmina balba»...; di M. Picone, Canto XIX, in G. Güntert e M. Picone (a cura di), Lectura Dantis Turicensis. Purgatorio, Firenze, Cesati, 2001, pp. 287-306, alle pp. 302-304 e B. Basile, s.v. Femmina, in ED, vol. II, 1970, pp. 835-836 che vengono a rappresentare il ventaglio delle proposte fatte.

18. Liber Scivias, ed. critica a cura di A. Führkötter e A. Carlevaris, Brepols, Turnhout, 1995: la visione, da cui le citazioni a testo, alle pp.576-577; se ne può leggere la traduzione moderna in Hildegarde de Bingen, Scivias. "Sache les voies» ou Livre des visions, présentation et traduction par P. Monat, Paris, Cerf, 1966.

19. Sul quale si veda Hildegard von Bingen, Wisse die Wege. Scivias. Nach den Originaltext des illuminierten rupertsberger Kodex ins deutsche übertragen und bearbeitet von Maura Böckeler, Salzburg, Otto Müller, 1954, con le 35 illustrazioni miniate accompagnate da commento e, ancora, L. Baillet, Les miniatures $d u$ "Scivias » de sainte Hildegarde conservé à la Bibliothèque de Wiesbaden, «Monuments et mémoires publiés par l'Académie des Inscriptions et Belles lettres» [Paris, Leroux], t. XIX, fasc. 1, 1911, pp. 49-149 e ora Hildegard von Bingen. Viaggio nelle immagini, a cura di S. Salvadori, Milano, Skira, 2019.

20. Si vedano E. Fenzi, Dio e Uomo nel cerchio della Trinità. Qualche nota ai versi finali della Commedia, «Letteratura \& Arte», $\mathrm{n}^{\circ} 16,2018$, pp. 23-52, part. pp. 32-34 e N. Tonelli, Gli angeli nei cerchi dell'Empireo, «Studi e problemi di critica testuale», vol. 90, nº 1, 2015, pp. 279-295.

21. Ricordo a questo proposito gli studi di Barucci e Rebuffat già citati.

22. Molto ampia la bibliografia sulle sirene e sul loro significato simbolico. Mi limito a segnalare qualche intervento recente: J. Arrouye, L'en-dessous et l'en-dessus de la mer, in C. ConnochieBourgne (a cura di), Mondes marins du Moyen Âge, Actes du $30^{\mathrm{e}}$ colloque du CUER MA ( 3,4 et 5 mars 2005), Aix-en-Provence, Publications de l'Université de Provence (PUP), 2006; I. Berti e F. Carlà, Mixanthropoi. Die mittelalterliche Rezeption antiker hybriden Kreaturen, in U. Rehm (a cura di), Mittelalterliche Mythenrezeption. Paradigmen und Paradigmenwechsel, Wien / Köln / Weimar, Böhlau, 2018, pp. 193-221; M. Bettini e L. Spina, Il mito delle Sirene. Immagini e racconti dalla Grecia a oggi, Torino, Einaudi, 2007.

\section{RIASSUNTI}

Balbuziente, guercia, storpia, senza mani e dilavata nel colore: ecco come appare la cosiddetta femmina balba nel sonno di Dante. Un essere orribile e ripugnante che però, nel corso del sogno narrato nel canto XIX del Purgatorio, subisce una straordinaria, incredibile metamorfosi: la nuova, bellissima immagine di donna inizia un canto di seduzione in cui si dichiara colma di piacere, tanto da saper appagare chiunque, una dolce sirena che ha tentato Ulisse nel suo viaggio. Anche questa è una delle figure femminili del Purgatorio, e non certo la meno significativa: 'falsa immagine di bene' creata dagli occhi desideranti del pellegrino. Da ripensare tenendo sullo sfondo la lunga riflessione sull'amore dei canti precedenti; da porre in relazione con la tradizione misogina, nei suoi rapporti con la letteratura medica e con la letteratura mistica, la femmina balba / dolce sirena è emblematica della natura perturbante del femminile, della doppiezza e ambiguità che è rintracciabile nella rappresentazione di molte altre donne della cantica.

Bègue, borgne, infirme, sans mains et aux couleurs délavées : c'est ainsi que la femme dite balba (bègue) apparaît dans le sommeil de Dante. Un être horrible et répugnant qui, cependant, au 
cours du rêve raconté dans le chant XIX du Purgatoire, subit une métamorphose extraordinaire, incroyable : la nouvelle image de très belle femme entame un chant de séduction dans lequel elle se déclare remplie de plaisir, au point de pouvoir satisfaire n'importe qui, telle la douce sirène qui tenta Ulysse dans son voyage. Elle aussi est l'une des figures féminines du Purgatoire, et certainement pas la moins significative: une "fausse image du bien», créée par les yeux désirants du pèlerin. Étant à reconsidérer dans le contexte de la longue réflexion sur l'amour dans les chants précédents, et à replacer par rapport à la tradition misogyne, dans ses rapports avec la littérature médicale et la littérature mystique, la femme balba / douce sirène est emblématique de la nature perturbatrice du féminin, de la duplicité et de l'ambiguïé que l'on retrouve dans la représentation de nombreuses autres femmes du cantique.

Stuttering, one-eyed, crippled, maimed, and wan: this is how the so-called femmina balba appears in Dante's sleep. A horrible and repulsive being who nonetheless, in the course of the dream narrated in Canto XIX of Purgatorio, undergoes an extraordinary, incredible metamorphosis: the new image of a beautiful woman begins a song of seduction in which she declares herself full of pleasure, thus able to satisfy anyone, a dolce sirena who led Ulysses into temptation. This too is one of the female figures in the Purgatorio, and certainly not the least significant: "falsa immagine di bene" created by the wishing eyes of the pilgrim. To be reinterpreted based on the long reflection on love of the previous Canti; to be related with the misogynist tradition, in its linkages with medical literature and with mystical literature, the femmina balba / dolce sirena is emblematic of the perturbing nature of the feminine, of the duplicity and ambiguity that can be traced in the representation of many other women of the Cantica.

\section{INDICE}

Keywords : love and medicine, misogyny, Hildegard of Bingen

Parole chiave : Purgatorio XIX, misoginia, Ildegarda di Bingen

Mots-clés : Purgatorio XIX, amour et médecine, Hildegarde de Bingen

\section{AUTORE}

\section{NATASCIA TONELLI}

Natascia Tonelli insegna Letteratura italiana all'Università degli Studi di Siena. Le sue ricerche si sono prevalentemente rivolte alla letteratura dei primi secoli (Cavalcanti, Dante, Petrarca, Boccaccio e lirica latina del Quattrocento), alla tradizione delle forme poetiche, alla poesia contemporanea (Zanzotto, Giudici, Raboni). I suoi ultimi libri sono Fisiologia della passione. Poesia d'amore e medicina da Cavalcanti a Boccaccio (Firenze, 2015), Per queste orme. Saggi sul Canzoniere (Pisa, 2016), Leggere il Canzoniere (Bologna, 2017). Condirige la rivista «Per leggere» e la collana «Didattica\&Letteratura» (Loescher). Fa parte del Comitato Nazionale per le celebrazioni del settecentenario dantesco e del Comitato scientifico dell'Ente Nazionale Giovanni Boccaccio. Natascia Tonelli enseigne la littérature italienne à l'Université de Sienne. Ses recherches ont principalement porté sur la littérature des premiers siècles (Cavalcanti, Dante, Pétrarque, Boccace et la poésie latine $\mathrm{du} \mathrm{Xv}^{\mathrm{e}}$ siècle), la tradition des formes poétiques et la poésie contemporaine (Zanzotto, Giudici, Raboni). Ses derniers livres sont Fisiologia della passione. Poesia d'amore e medicina da Cavalcanti a Boccaccio (Florence, 2015), Per queste orme. Saggi sul Canzoniere (Pise, 2016), Leggere il Canzoniere (Bologne, 2017). Elle co-dirige la revue Per leggere et la collection 
« Didattica\&Letteratura » (Loescher). Elle est membre du comité national pour les célébrations du $700^{e}$ anniversaire de Dante et du comité scientifique de l'Ente Nazionale Giovanni Boccaccio. natascia.tonelli@unisi.it 